\title{
Soil biofilms: microbial interactions, challenges, and advanced techniques for ex-situ characterization
}

\author{
Peng Cai ${ }^{1}{ }^{*}$, Xiaojie Sun ${ }^{1}$, Yichao $\mathrm{Wu}^{1}$, Chunhui Gao ${ }^{1}$, Monika Mortimer ${ }^{2}$, Patricia A. Holden ${ }^{2}$, \\ Marc Redmile-Gordon ${ }^{3}$, Qiaoyun Huang ${ }^{1}$ \\ 1 State Key Laboratory of Agricultural Microbiology, College of Resources and Environment, Huazhong Agricultural University, \\ Wuhan 430070, China \\ 2 Bren School of Environmental Science and Management and Earth Research Institute and University of California Center for the \\ Environmental Implications of Nanotechnology (UC CEIN), University of California, Santa Barbara, CA 93106, USA \\ 3 Department of Environmental Horticulture, Royal Horticultural Society, Wisley, Surrey, GU23 6QB, UK
}

ARTICLE INFO

\section{Article history:}

Received January 22, 2019

Revised March 28, 2019

Accepted April 18, 2019

Keywords:

Soil microbiome

Soil biofilm

Microbial interactions

Micro-scale environments

Biofilm cultivation devices

Microfluidic techniques

\begin{abstract}
A B S TR A C T
Soil is inhabited by a myriad of microorganisms, many of which can form supracellular structures, called biofilms, comprised of surface-associated microbial cells embedded in hydrated extracellular polymeric substance that facilitates adhesion and survival. Biofilms enable intensive inter- and intra-species interactions that can increase the degradation efficiency of soil organic matter and materials commonly regarded as toxins. Here, we first discuss organization, dynamics and properties of soil biofilms in the context of traditional approaches to probe the soil microbiome. Social interactions among bacteria, such as cooperation and competition, are discussed. We also summarize different biofilm cultivation devices in combination with optics and fluorescence microscopes as well as sequencing techniques for the study of soil biofilms. Microfluidic platforms, which can be applied to mimic the complex soil environment and study microbial behaviors at the microscale with highthroughput screening and novel measurements, are also highlighted. This review aims to highlight soil biofilm research in order to expand the current limited knowledge about soil microbiomes which until now has mostly ignored biofilms as a dominant growth form.
\end{abstract}

() Higher Education Press 2019

\section{Characteristics of soil biofilms}

Soil provides numerous available surfaces for formation of multispecies biofilms in which bacteria colonize microhabitats, occupying less than $1 \%$ of total soil volume and merely $10^{-6} \%$ of the soil surface area (Young and Crawford, 2004; Young et al., 2008). Biofilms in soil consist of diverse species embedded in a self-produced matrix of extracellular polymeric substance (EPS), attaching to soil particles or biotic surfaces

\footnotetext{
* Corresponding author

E-mail address: cp@mail.hzau.edu.cn (P. Cai)
}

such as roots and fungal hyphae and decomposing organic material (Burmolle et al., 2012; Flemming and Wuertz, 2019). Although soil environments are complex and dynamic, biofilms can stabilize the conditions surrounding bacteria, providing protection against predation, desiccation and exposure to antibiotics, while improving the availability of nutrients and oxygen, and providing a niche for horizontal gene transfer (Sørensen et al., 2005; Madsen et al., 2012).

\subsection{The soil microbiome and biofilms}

Microorganisms, the earliest forms of life on earth, determine the direction and process of the evolution of earth, and nurture human civilization (Dodd et al., 2017). The soil microbiome, 
which includes all microorganisms found in any given soil, is known as the engine which drives many key biogeochemical cycles. The microbiome maintains nutrient cycling, soil fertility and is implicated in soil carbon storage (Liang and Balser, 2011), with a direct or indirect effect on the health of plants and animals in terrestrial ecosystems (Ahmad et al., 2017; Fierer, 2017).

Early methods of microbiological research involved adding an inoculum and culture medium to a flask, and shaking under specific conditions. This allows microbial cells to acquire enough nutrients to grow and reproduce in a planktonic state. However, microorganisms in the natural environment frequently exist as surface-attached structures termed biofilms (Flemming and Wingender, 2010; McDougald et al., 2011; Flemming et al., 2016). Biofilms generally colonize carbon rich surfaces of soil media such as plant roots and clay minerals, and secrete EPS to form aggregates (Ma et al., 2017; Nunan, 2017; Cai et al., 2018). The multicellular aggregates formed by soil microbes are known as soil biofilms, or unsaturated biofilms, owing to low water availability as the distinguishing environmental factor of the local environment (Holden, 2001).

\subsection{Organization of soil microbial communities}

When studying the soil microbiome, some researchers consider microbes as a whole and assume that they are closely interacting with each other. However, this is not how microbes inhabit the soil. Heterogeneity and diversity are inherent properties of soil habitats (Kuzyakov and Blagodatskaya, 2015). The spatial arrangement of soil creates numerous micro-habitats and provides simultaneous living space for microorganisms that occupy contrasting niches. In recent years, sectioning of intact soil has shown that soil microbes exist in the form of individual micro-aggregates (Nunan, 2017). These micro-aggregates are small in size, with only a few hundred cells and limited number of species, and separated from other micro-aggregates by several tens of micrometers (Raynaud and Nunan, 2014). Thus in unsaturated conditions, a single aggregate may contain a myriad of different microbial communities, each composed of thousands of individuals (Vos et al., 2013; Rillig et al., 2017). These soil microbial communities lack connectivity with each other, and thus resemble biogeographical islets, which contribute to spatial and biochemical heterogeneity at the micro-scale (Fig. 1A).

A

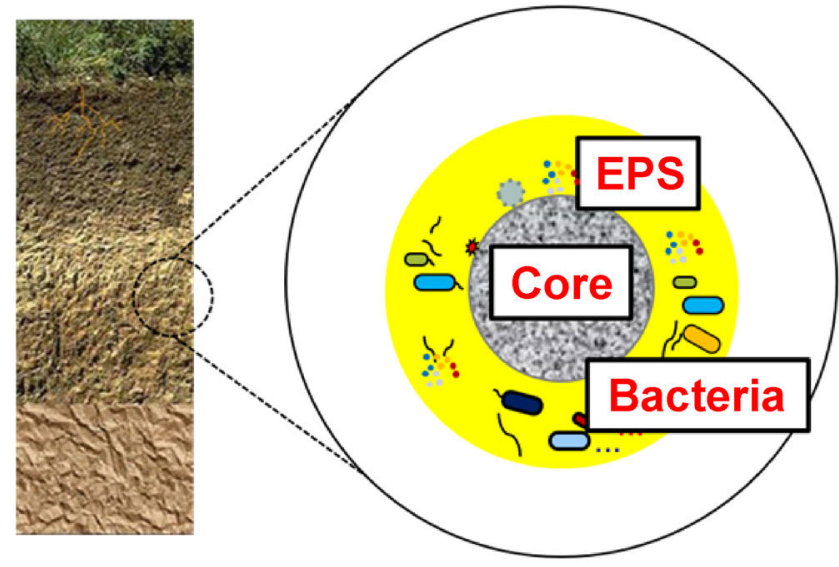

B

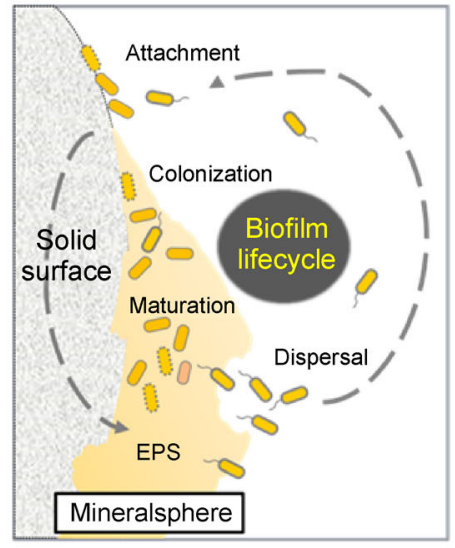

C

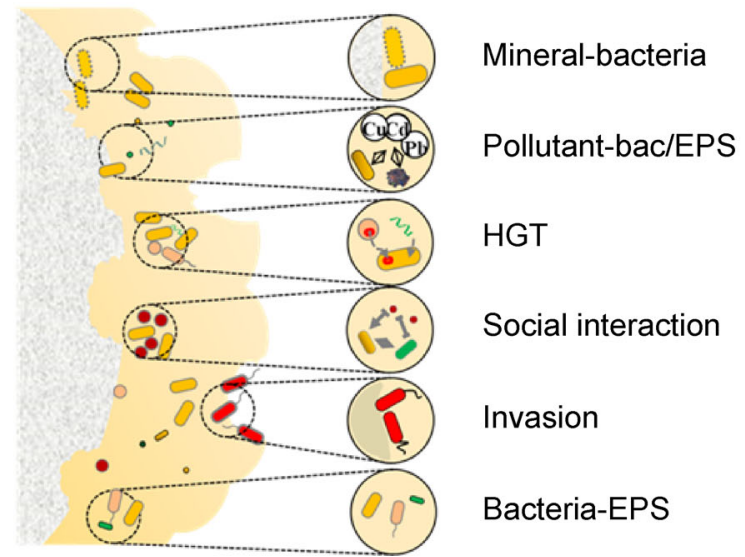

Fig. 1 Soil biofilm and its unique properties. (A) The soil biofilm is organized at the micro-scale. (B) The life cycle of a soil biofilm consists of four phrases: attachment, colonization, maturation, and dispersal. (C) The unique properties of soil biofilms: rods with different colors represent various bacteria; extracellular polymeric substances (EPS); horizontal gene transfer (HGT). 
1.3 Soil biofilms are not static but dynamic at spatial and temporal scales

The life cycle of a soil biofilm can be divided into several stages: i) attachment onto solid surfaces (such as minerals and plant roots), ii) colonization of these surfaces, iii) development, iv) maturation and v) cell dispersal (Fig. 1B). Attachment is a physicochemical process, subject to the interplay of surface charge, dissolved ions, and fluid mechanics (Cai et al., 2013). When microorganisms sense the surface, profound physiological changes will occur at the interface which prompts colonization (O'Toole and Wong, 2016). During the development of a biofilm, the physiology of cells diverges from that of planktonic counterparts, producing a biochemically distinct phenotype and physical form. At maturation the biofilm structure and composition are relatively stable. However, with the depletion of nutrients or external disturbances, the cells within can respond quickly - releasing enzymes capable of dispersing the biofilm and triggering the dispersal of cells (Oppenheimer-Shaanan et al., 2013). During biofilm dispersal, cells can detach from the biofilm matrix in active or passive ways. These detached cells may colonize another site and start a new biofilm cycle (Fig. 1B).

\subsection{Unique properties of soil biofilms}

When compared to their planktonic counterparts, biofilmassociated bacteria generally have strong fitness advantages (Kragh et al., 2016). Robust, ecologically important, biogeochemical processes occur in the small volume of these biofilms, forming soil hotspots (Kuzyakov and Blagodatskaya, 2015). For example, a biofilm of Bradyrhizobium japonicum SEMIA 5019-Penicillium spp. increased the mineralization of organic nitrogen and phosphorus in a soil, and showed higher nitrogenase activity even under high $\mathrm{NO}_{3}{ }^{-}$concentrations compared to soils containing planktonic members of the same species (Seneviratne and Jayasinghearachchi, 2005). Species interactions across major taxonomic groups within biofilms also reveal beneficial impacts on soil aggregation (Lehmann et al., 2017). In addition, EPS accounting for $80 \%$ of biofilm's dry mass (Chenu, 1993) can provide numerous beneficial functions in soils such as protecting microorganisms against biotic and abiotic stresses (Or et al., 2007). For example, EPS can store 15 to 20 times its own weight in water (Chenu and Roberson, 1996), thus significantly increase the water holding capacity of soils (Adessi et al., 2018). EPS also contributes to the formation and stability of soil aggregates (Bezzate et al., 2000; Büks and Kaupenjohann, 2016), in which the magnitude of the effect is largely dependent on the characteristics of EPS (Chenu and Cosentino, 2011) and soil physico-chemical properties (Martin and Aldrich, 1955).

For bacterial biofilms and their interactions, attention is usually to biotechnological applications and clinically relevant fields (Perez-Garcia et al., 2016; Kenny and Balskus, 2018; Magana et al., 2018), while the importance of soil biofilms has largely been ignored. From the perspective of soil microbiology, research in different fields are needed to lay the foundation for researching soil biofilms and to drive this field into a new era.

\section{Microbial interactions in soil biofilms}

Cells in biofilms are known to interact with each other and exhibit many social activities due to their close proximity, which inspired the introduction of the anthropomorphic term "sociomicrobiology" (Parsek and Greenberg, 2005). These social behaviors involve inter- and intra-specific interactions, the exchange of metabolic products and intercellular signaling (Burmølle et al., 2014). Due to the diversity of microbes in biofilm communities, the interactions in biofilms are always diverse and dynamic, and play important roles in the formation and function of soil biofilms.

\subsection{Cooperative interactions}

Microbes in nature tend not to exist in isolation and can cooperate to build a biofilm (Faust and Raes, 2012). The formation of biofilms is conducive to protection against environmental stresses, implying that microbes can cooperate to form multi-species biofilms which jointly cope with unfavorable living conditions. For example, Burmølle et al. (2007) found that Pseudomonas putida SB5 could promote biofilm formation by Chryseobacterium sp. SB9, resulting in a dualspecies biofilm which was also found to exhibit strain-specific properties. Another study has also shown significant synergetic interactions in a four-species biofilm containing Stenotrophomonas rhizophila, Xanthomonas retroflexus, Microbacterium oxydans and Paenibacillus amylolyticus, with $X$. retroflexus playing a dominant role and the other strains a supplementary role in the formation of the multi-species biofilm (Ren et al., 2015).

Bacteria in biofilms are in close proximity to one another, which is a prerequisite for the trade of metabolites between cells. Metabolites containing amino acids produced by other species are frequently used by bacteria for growth in laboratory-based settings or natural environments, which is ecologically termed as cross-feeding (Pande et al., 2016). Most microorganisms in a biofilm are auxotrophic, and more than $98 \%$ of the microorganisms sequenced so far lack essential pathways or key genes for the synthesis of amino acids (Zengler and Zaramela, 2018) which suggests that the trade of metabolites among different species in biofilms is very important for survival. For example, metabolic cooperation can occur when ammonia is supplied to ammonia-oxidizers by nitrite-oxidizing bacteria, such as Nitrospira moscoviensis (Flemming et al., 2016). Cross-feeding can even be observed in inter-domain interactions, e.g. with Streptococcus mutans and Candida albicans. Both are found together in biofilms from early childhood caries, with $C$. albicans benefiting from lactic acid produced by S. mutans (Raja et al., 2010; Metwalli et al., 2013; Sztajer et al., 2014).

The increasing tolerance of biofilms to antibiotics and surfactants is also the consequence of mutually beneficial 
interactions (Harrison et al., 2007; Lee et al., 2014; Olsen, 2015). Moreover, bacteria can cooperate in contaminant degradation, such as with the herbicide linuron and 2,4dichlorophenoxyacetic acid (Aspray et al., 2005; Breugelmans et al., 2008; Flemming, 2010). Overall, co-metabolism in soil biofilms is suspected to play a vital role in contaminant degradation, nutrient cycling, and organic matter turnover (Fig. 1C).

\subsection{Intra- and inter-species competition}

Viewed externally, cooperation would appear to be the main ecological interaction among biofilm organisms, but competition also occurs. On one hand, individuals, or populations, may strive for advantage in terms of space or resource utilization. On the other hand, the micro-environment afforded by the soil biofilm is still vulnerable to external disturbances which may destroy the foundation of this stability resulting in shuffling of the community structure, which we summarize as post-disturbance biofilm competition.

The exploitation of contrasting niches in a biofilm is a community consequence of evolution constrained by time and space. For example, with anaerobic biofilms developed on the surfaces of sewer pipes, sulfate-reducing bacteria (SRB) and methanogenic archaea (MA) compete with each other for substrates including organic matter, acetate and hydrogen. SRB were dominant in the initial phase of biofilm formation but, on maturation, methanogenic colonizers were better adapted and replaced the SRB (Auguet et al., 2015). Competition pressure for nutrients is particularly high among phylogenetically similar strains in soil, marine and other natural environments (Hawlena et al., 2010; Schoustra et al., 2012; Patin et al., 2016). Researchers measured the pairwise interactions among 64 Streptomyces strains isolated from several individual grains of a soil sample and found reciprocity between strains, which means if a strain inhibits or promotes the growth of a second strain, the second strain is likely to affect the first strain in a similar manner (Vetsigian et al., 2011). Such reciprocity is further enhanced in strains derived from the same soil grain, suggesting that it may be a property of coexisting communities. Other studies indicate that an increase in biofilm formation is a common adaptive response to long-term coexistence (Madsen et al., 2016). A pairwise interaction assay of 67 bacterial species also showed that antagonism is most likely to occur among closely related species, and that the more similar the strains are metabolically, the higher the probability of antagonism (Russel et al., 2017). Given the factors above, it is clear that both inter- and intra-specific diversity are important metrics for understanding and predicting biofilm behavior.

To gain competitive advantage, a bacterial taxa may rely on many different approaches, two examples being i) inhibiting the growth of other taxa by nutrient competition through the secretion of siderophores and ii) killing other competitors by secreting broad-spectrum antibiotics (Hibbing et al., 2010; Gao et al., 2019). These two approaches have been described as "pirate" and "killer," respectively (Szamosvári et al., 2018). For example, bacteria can produce antibacterial compounds such as bacteriocins, antibiotics and surfactants to inhibit the growth of adjacent taxa, resulting in competition between cells. Additionally, Bacillus spp. and Pseudomonas spp., which have been referred to as biocontrol bacteria, produce 2,4-diacetylphloroglucinol which can inhibit the growth of plant pathogens (Raaijmakers and Mazzola, 2012). The secretion system ('Type VI') of some pathogens like Pseudomonas aeruginosa can transport the virulent proteins into neighboring cells, which can inhibit the growth of and even kill these cells (Basler et al., 2013; Nadell et al., 2016).

In natural environments, high order inter- and intra-taxa interactions are central properties of ecological networks because of high diversity and rapid co-evolution of microbes. Current studies mainly focus on the relationship between biodiversity and ecosystem functioning by analyzing multispecies mixtures that combine taxa chosen at random (Foster and Bell, 2012). There is a rapid and significant increase in productivity with more taxa added, especially for ecologically indigenous taxa, which may explain the observed rise in the total population size when the diversity of taxa increases (Freilich et al., 2011; Gravel et al., 2011).

\section{Ex-situ techniques in soil biofilm research}

3.1 Biofilm cultivation devices, microscopy and sequencing techniques

Direct characterization of biofilms in soil is incredibly challenging due to spatial heterogeneity within any single soil aggregate. The opacity of the soil matrix makes it almost impossible to visualize soil biofilms using microscopy. Therefore, studying biofilm development processes ex-situ is advantageous to further our understanding of the functional capacity of a sampled soil community. Various constructs can be used as biofilm cultivation devices, such as microtiter plates, drip flow biofilm reactors, and rotary biofilm reactors (Fig. 2). The microtiter-based cultivation approach provides a platform for high-throughput screening of multispecies biofilm formation and can be used as a standard procedure for evaluating interspecies interactions in defined microbial communities (Burmølle et al., 2007; Ren et al., 2014).

Biofilm cultivation devices can yield biofilms that can be analyzed using optical, including fluorescence, microscopes such as confocal laser scanning microscopy (CLSM) to enable visualization of biofilm structure. Fluorescent protein tagging and fluorescence in situ hybridization (FISH) allow the visualization of spatial arrangements of community members (Liu et al., 2017, 2018). Fluorescent dye staining also enables the analysis of cell viability, EPS composition and metabolic activities (Guilbaud et al., 2015; Azeredo et al., 2017).

The rapid development of sequencing techniques can further reveal phylogenetic composition and gene expression during biofilm formation (Sztajer et al., 2014; Trejo-Hernández et al., 2014; Maeda et al., 2015; Liang et al., 2017). 


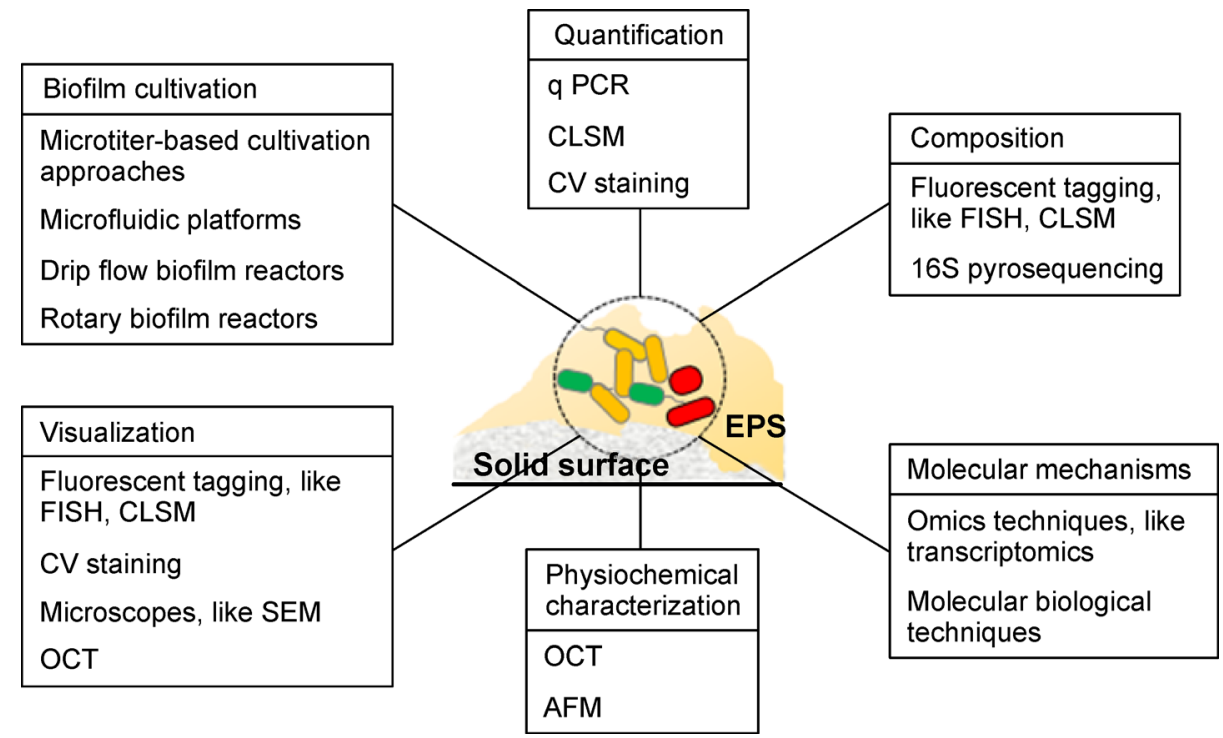

Fig. 2 Cultivation systems and analytical techniques for ex-situ biofilm research. Rods with different colors represent bacteria in biofilm. FISH, fluorescence in situ hybridization; CLSM, confocal laser scanning microscopy; SEM, scanning electron microscope; CV staining, crystal violet staining; OCT, optical coherence tomography; AFM, atomic force microscope.

Comparative gene expression analysis of the $X$. retroflexus transcriptome in single-species biofilm and dual- and fourspecies biofilm with $S$. rhizophila, $M$. oxydans and $P$. amylolyticus revealed complex interdependent interaction patterns in the multispecies biofilms and the significant role of cross feeding in synergistic biofilm production (Hansen et al., 2017). As a further example, four taxa, including Pseudomonas fluorescens, Bacillus subtilis, Brevundimonas and Pedobacter typically co-exist in soil. In the absence of an exogenous carbon source, the latter three taxa, especially $B$. subtilis, will inhibit the growth of $P$. fluorescens. Transcriptome analysis revealed that $P$. fluorescens varies significantly at the transcriptional level in response to different taxa, with both Brevundimonas and Pedobacter promoting the secretion of a broad-spectrum antibiotic from $P$. fluorescens (Garbeva et al., 2011). Moreover, metagenomics and 16S rRNA gene amplicon sequencing have provided great insight into the structures and dynamics of soil microbial communities (Faust and Raes, 2012). The networks obtained from these studies based on microbial operational taxonomic units (OTUs) can be used for predicting bacterial interactions in natural environments from oceans to the human microbiome (Chaffron et al., 2010; Methé et al., 2012).

\subsection{Microfluidics offer novel experimental opportunities}

Microfluidics techniques have been widely applied in biomedicine in recent decades. The precise control of structure and fluids at the microscopic scale offers a new approach to simulate a heterogeneous soil environment. Coupled with other modern toolkits, it has great potential to contribute to the study of microbial interactions in soil biofilms (Aleklett et al., 2018). Currently, existing microfluidic chips (devices) can be divided into three categories viz. continuous flow devices, paper based devices, and digital microfluidic devices (Liu and Walther-Antonio, 2017). Continuous flow devices are the simplest and sometimes referred to as flow cells (Azeredo et al., 2017). The capture device in the microfluidic system is usually an optical microscope (conventional, fluorescence, or confocal laser). To track single-cells, the microscope needs to perform autofocus and focus shift compensation or collect high-resolution image data with a confocal microscope. The spatial location, size, orientation, biomass, etc. are then obtained by data and image processing (Oliveira et al., 2015).

While techniques such as flow cells and optical coherence tomography (OCT; which can image fluorescently labeled biofilms) are not suitable for high-throughput studies, they can provide valuable qualitative and quantitative information regarding spatial structure. Moreover, since current omics techniques normally require the disruption of soil spatial structure, they can only provide raw repertoires of taxa and genes, and fail to provide information about biological interactions between organisms at the microscale $(\sim 100 \mu \mathrm{m})$ where most effects on population dynamics and composition occur (Cordero and Datta, 2016). Microfluidic platforms can be applied to mimic the soil-environment and study microbial behavior at the microscale, including simulating physical heterogeneity, creating chemical gradients and patches, manipulating microbial interactions, and studying rhizosphere interactions (For details see Garcia-Cordero and Maerkl, 2014; Aleklett et al., 2018).

In combination with advanced imaging techniques and appropriate fluorescence tags, microfluidic platforms can provide insight into the bacterial interactions in soil at the microscale. With a modified microfluidic-based device enabling direct imaging of root-bacterial interactions in real time, Massalha et al. (2017) monitored biofilm formation of a fluorescently labeled $B$. subtilis colonizing the root of 
Soil biofilms: microbial interactions, challenges, and advanced techniques for ex-situ characterization

Arabidopsis thaliana and interacting with fluorescently tagged cells of Escherichia coli in the rhizosphere. Real-time imaging of these two strains showed that bacteria compete for root surface colonization. In addition, microfluidic devices can also be utilized to control spatial structure and chemical communication in a community, which may facilitate the understanding of how communities of microbes interact and perform community-level functions in natural ecosystems and how the diversity of microbial communities is maintained at the microscale (Rusconi et al., 2014; Liu et al., 2016). In a community which consisted of three soil bacterial taxa (Azotobacter vinelandii, Bacillus licheniformis, and Paenibacillus curdlanolyticus) with syntrophic interactions, each taxa was localized by a microfluidic device into an individual culture well separated from a microfluidic communication channel by a nano-porous membrane. The maintenance of these taxa simultaneously under these conditions demonstrated that a defined microscale spatial structure is both necessary and sufficient for the stable coexistence of interacting bacterial species in a community (Kim et al., 2008).

\section{Perspective}

Soil biofilms offer advantages for microbes living within them. Studies on the mechanisms of soil biofilm development, organization and microbial interactions will provide useful information for the management of soil microbes for desired outcomes: be they environmental, agricultural, or biotechnological. However, the spatial and chemical heterogeneity of soil conditions make it difficult to mimic biofilms at the scale of soil particles and also almost impossible to observe biofilms in situ. Additionally, the EPS matrix provides different localized habitats at the microscale with dynamic gradients of oxygen, $\mathrm{pH}$ and nutrients (Flemming et al., 2016), which further increases the difficulty of replicating soil biofilm conditions. Microfluidics devices combined with appropriate fluorescent labels and imaging techniques can provide unique insights into microscale ecology of this complex microenvironment and are therefore likely to enhance our understanding of this momentous field of research (Massalha et al., 2017). In the future, such devices should be designed to represent variations in conditions that are unique to the soil environment, namely oscillating wet and dry phases that occur naturally with water infiltration followed by evaporation events. Given that microaggregate isolation is facilitated by disconnected water films that develop with soil drying, and given that disconnectedness is so profoundly influential in structuring biofilm diversity and function, aspiring to allow for microfluidic devices to operation under controllable unsaturated conditions will allow for approaching a more realistic understanding of soil biofilms.

\section{Acknowledgments}

This work was supported by the National Natural Science Foundation of China (41877029), the National Basic Research
Program of China (2016YFD0800206) and the Fundamental Research Funds for the Central Universities (2662017JC008).

\section{References}

Adessi, A., de Carvalho, R.C., De Philippis, R., Branquinho, C., da Silva, J.M., 2018. Microbial extracellular polymeric substances improve water retention in dryland biological soil crusts. Soil Biology \& Biochemistry 116, 67-69.

Ahmad, I., Khan, M.S., Altaf, M.M., Qais, F.A., Ansari, F.A., Rumbaugh, K.P., 2017. Biofilms: an overview of their significance in plant and soil health. In: Ahmad, I., Husain, F.M. (Eds.), Biofilms in Plant and Soil Health. John Wiley \& Sons, Ltd, Chichester, UK, pp. 1-25.

Aleklett, K., Kiers, E.T., Ohlsson, P., Shimizu, T.S., Caldas, V.E., Hammer, E.C., 2018. Build your own soil: exploring microfluidics to create microbial habitat structures. ISME Journal 12, 312-319.

Aspray, T.J., Hansen, S.K., Burns, R.G., 2005. A soil-based microbial biofilm exposed to 2,4-D: bacterial community development and establishment of conjugative plasmid pJP4. FEMS Microbiology Ecology 54, 317-327.

Auguet, O., Pijuan, M., Batista, J., Borrego, C.M., Gutierrez, O., 2015. Changes in microbial biofilm communities during colonization of sewer systems. Applied and Environmental Microbiology 81, 7271-7280.

Azeredo, J., Azevedo, N.F., Briandet, R., Cerca, N., Coenye, T., Costa, A.R., Desvaux, M., Di Bonaventura, G., Hébraud, M., Jaglic, Z., Kačániová, M., Knøchel, S., Lourenço, A., Mergulhão, F., Meyer, R.L., Nychas, G., Simões, M., Tresse, O., Sternberg, C., 2017. Critical review on biofilm methods. Critical Reviews in Microbiology 43, 313-351.

Basler, M., Ho, B.T., Mekalanos, J.J., 2013. Tit-for-tat: type VI secretion system counterattack during bacterial cell-cell interactions. Cell 152, 884-894.

Bezzate, S., Aymerich, S., Chambert, R., Czarnes, S., Berge, O., Heulin, T., 2000. Disruption of the Paenibacillus polymyxa levansucrase gene impairs its ability to aggregate soil in the wheat rhizosphere. Environmental Microbiology 2, 333-342.

Breugelmans, P., Barken, K.B., Tolker-Nielsen, T., Hofkens, J., Dejonghe, W., Springael, D., 2008. Architecture and spatial organization in a triple-species bacterial biofilm synergistically degrading the phenylurea herbicide linuron. FEMS Microbiology Ecology 64, 271-282.

Büks, F., Kaupenjohann, M., 2016. Enzymatic biofilm digestion in soil aggregates facilitates the release of particulate organic matter by sonication. Soil (Göttingen) 2, 499-509.

Burmølle, M., Hansen, L.H., Sørensen, S.J., 2007. Establishment and early succession of a multispecies biofilm composed of soil bacteria. Microbial Ecology 54, 352-362.

Burmolle, M., Kjoller, A., Sorensen, S.J., 2012. An invisible workforce: biofilms in the soil. In: Gavin L., Gillian L., (Eds.). Microbial Biofilm: Current Research and Applications. Caister Academic Press, pp. 61-71.

Burmølle, M., Ren, D., Bjarnsholt, T., Sørensen, S.J., 2014. Interactions in multispecies biofilms: do they actually matter? 
Trends in Microbiology 22, 84-91.

Cai, P., Huang, Q., Walker, S.L., 2013. Deposition and survival of Escherichia coli $\mathrm{O} 157 \mathrm{H} 7$ on clay minerals in a parallel plate flow system. Environmental Science \& Technology 47, 1896-1903.

Cai, P., Liu, X., Ji, D., Yang, S., Walker, S.L., Wu, Y., Gao, C., Huang, Q., 2018. Impact of soil clay minerals on growth, biofilm formation, and virulence gene expression of Escherichia coli O157:H7. Environmental Pollution 243, 953-960.

Chaffron, S., Rehrauer, H., Pernthaler, J., von Mering, C., 2010. A global network of coexisting microbes from environmental and whole-genome sequence data. Genome Research 20, 947-959.

Chenu, C., 1993. Clay polysaccharide or sand polysaccharide associations as models for the interface between microorganisms and soil water related properties and microstructure. Geoderma 56, 143-156.

Chenu, C., Cosentino, D., 2011. Microbial regulation of soil structural dynamics. In: Ritz, K., Young, I., (Eds.), The architecture and biology and soils: life in inner space. CAB International, London, pp. 37-70.

Chenu, C., Roberson, E.B., 1996. Diffusion of glucose in microbial extracellular polysaccharide as affected by water potential. Soil Biology \& Biochemistry 28, 877-884.

Cordero, O.X., Datta, M.S., 2016. Microbial interactions and community assembly at microscales. Current Opinion in Microbiology 31 , 227-234.

Dodd, M.S., Papineau, D., Grenne, T., Slack, J.F., Rittner, M., Pirajno, F., O'Neil, J., Little, C.T., 2017. Evidence for early life in Earth's oldest hydrothermal vent precipitates. Nature 543, 60-64.

Holden, 2001, Biofilms in unsaturated environments. In: Doyle, R.J., ed., Methods of Enzymology. Academic Press, San Diego, California, pp. 125-143.

Faust, K., Raes, J., 2012. Microbial interactions: from networks to models. Nature Reviews. Microbiology 10, 538-550.

Fierer, N., 2017. Embracing the unknown: disentangling the complexities of the soil microbiome. Nature Reviews. Microbiology 15, 579-590.

Flemming, H.C., 2010. Biodeterioration of synthetic materials-a brief review. Materials and Corrosion 61, 986-992.

Flemming, H.C., Wingender, J., 2010. The biofilm matrix. Nature Reviews. Microbiology 8, 623-633.

Flemming, H.C., Wingender, J., Szewzyk, U., Steinberg, P., Rice, S. A., Kjelleberg, S., 2016. Biofilms: an emergent form of bacterial life. Nature Reviews. Microbiology 14, 563-575.

Flemming, H.C., Wuertz, S., 2019. Bacteria and archaea on Earth and their abundance in biofilms. Nature Reviews. Microbiology 17 , 247-260.

Foster, K.R., Bell, T., 2012. Competition, not cooperation, dominates interactions among culturable microbial species. Current Biology 22, 1845-1850.

Freilich, S., Zarecki, R., Eilam, O., Segal, E.S., Henry, C.S., Kupiec, M., Gophna, U., Sharan, R., Ruppin, E., 2011. Competitive and cooperative metabolic interactions in bacterial communities. Nature Communications 2, 589.

Gao, C.H., Zhang, M., Wu, Y., Huang, Q., Cai, P., 2019. Divergent influence to a pathogen invader by resident bacteria with different social interactions. Microbial Ecology 77, 76-86.
Garbeva, P., Silby, M.W., Raaijmakers, J.M., Levy, S.B., Boer, W., 2011. Transcriptional and antagonistic responses of Pseudomonas fluorescens Pf0-1 to phylogenetically different bacterial competitors. ISME Journal 5, 973-985.

Garcia-Cordero, J.L., Maerkl, S.J., 2014. A 1024-sample serum analyzer chip for cancer diagnostics. Lab on a Chip 14, 2642 2650.

Gravel, D., Bell, T., Barbera, C., Bouvier, T., Pommier, T., Venail, P., Mouquet, N., 2011. Experimental niche evolution alters the strength of the diversity-productivity relationship. Nature 469 , 89-92.

Guilbaud, M., Piveteau, P., Desvaux, M., Brisse, S., Briandet, R., 2015. Exploring the diversity of Listeria monocytogenes biofilm architecture by high-throughput confocal laser scanning microscopy and the predominance of the honeycomb-like morphotype. Applied and Environmental Microbiology 81, 1813-1819.

Hansen, L.B.S., Ren, D., Burmølle, M., Sørensen, S.J., 2017. Distinct gene expression profile of Xanthomonas retroflexus engaged in synergistic multispecies biofilm formation. ISME Journal 11, 300303.

Harrison, J.J., Ceri, H., Turner, R.J., 2007. Multimetal resistance and tolerance in microbial biofilms. Nature Reviews. Microbiology 5 , 928-938.

Hawlena, H., Bashey, F., Lively, C.M., 2010. The evolution of spite: population structure and bacteriocin-mediated antagonism in two natural populations of Xenorhabdus bacteria. Evolution 64, 31983204.

Hibbing, M.E., Fuqua, C., Parsek, M.R., Peterson, S.B., 2010. Bacterial competition: surviving and thriving in the microbial jungle. Nature Reviews. Microbiology 8, 15-25.

Kenny, D.J., Balskus, E.P., 2018. Engineering chemical interactions in microbial communities. Chemical Society Reviews 47, 17051729.

Kim, H.J., Boedicker, J.Q., Choi, J.W., Ismagilov, R.F., 2008. Defined spatial structure stabilizes a synthetic multispecies bacterial community. Proceedings of the National Academy of Sciences of the United States of America 105, 18188-18193.

Kragh, K.N., Hutchison, J.B., Melaugh, G., Rodesney, C., Roberts, A. E.L., Irie, Y., Jensen, P.Ø., Diggle, S.P., Allen, R.J., Gordon, V., Bjarnsholt, T., 2016. Role of multicellular aggregates in biofilm formation. mBio 7, e00237-e16.

Kuzyakov, Y., Blagodatskaya, E., 2015. Microbial hotspots and hot moments in soil: Concept \& review. Soil Biology \& Biochemistry 83, 184-199.

Lee, K.W.K., Periasamy, S., Mukherjee, M., Xie, C., Kjelleberg, S., Rice, S.A., 2014. Biofilm development and enhanced stress resistance of a model, mixed-species community biofilm. ISME Journal 8, 894-907.

Lehmann, A., Zheng, W., Rillig, M.C., 2017. Soil biota contributions to soil aggregation. Nature Ecology \& Evolution 1, 1828-1835.

Liang, C., Balser, T.C., 2011. Microbial production of recalcitrant organic matter in global soils: implications for productivity and climate policy. Nature Reviews. Microbiology 9, 75, author reply 75.

Liang, J., Bai, Y., Men, Y., Qu, J., 2017. Microbe-microbe interactions trigger Mn(II)-oxidizing gene expression. ISME Journal 11, 67-77. 
Liu, W., Røder, H.L., Madsen, J.S., Bjarnsholt, T., Sørensen, S.J., Burmølle, M., 2016. Interspecific bacterial interactions are reflected in multispecies biofilm spatial organization. Frontiers in Microbiology 7, 1366.

Liu, W., Russel, J., Burmølle, M., Sørensen, S.J., Madsen, J.S., 2018. Micro-scale intermixing: a requisite for stable and synergistic coestablishment in a four-species biofilm. ISME Journal 12, 19401951.

Liu, W., Russel, J., Røder, H.L., Madsen, J.S., Burmølle, M., Sørensen, S.J., 2017. Low-abundant species facilitates specific spatial organization that promotes multispecies biofilm formation. Environmental Microbiology 19, 2893-2905.

Liu, Y., Walther-Antonio, M., 2017. Microfluidics: a new tool for microbial single cell analyses in human microbiome studies. Biomicrofluidics 11, 061501.

Ma, W.T., Peng, D.H., Walker S.L., Cao, B., Gao, C.H., Huang, Q.Y., Cai, P., 2017. Bacillus subtilis biofilm development in the presence of soil clay minerals and iron oxides. npj Biofilms and Microbiomes 3:4.

Madsen, J.S., Burmølle, M., Hansen, L.H., Sørensen, S.J., 2012. The interconnection between biofilm formation and horizontal gene transfer. FEMS Immunology and Medical Microbiology 65, 183195.

Madsen, J.S., Røder, H.L., Russel, J., Sørensen, H., Burmølle, M., Sørensen, S.J., 2016. Coexistence facilitates interspecific biofilm formation in complex microbial communities. Environmental Microbiology 18, 2565-2574.

Maeda, K., Nagata, H., Ojima, M., Amano, A., 2015. Proteomic and transcriptional analysis of interaction between oral microbiota Porphyromonas gingivalis and Streptococcus oralis. Journal of Proteome Research 14, 82-94.

Magana, M., Sereti, C., loannidis, A., Mitchell, C.A., Ball, A.R., Magiorkinis, E., Chatzipanagiotou, S., Hamblin, M.R., Hadjifrangiskou, M., Tegos, G.P., 2018. Options and limitations in clinical investigation of bacterial biofilms. Clinical Microbiology Reviews 31, e00084-e16.

Martin, J.P., Aldrich, D.G., 1955. Influence of soil exchangeable cation ratios on the aggregating effects of natural and synthetic soil conditioners. Soil Science Society of America Journal 19, 50-54.

Massalha, H., Korenblum, E., Malitsky, S., Shapiro, O.H., Aharoni, A., 2017. Live imaging of root-bacteria interactions in a microfluidics setup. Proceedings of the National Academy of Sciences of the United States of America 114, 4549-4554.

McDougald, D., Rice, S.A., Barraud, N., Steinberg, P.D., Kjelleberg, S., 2011. Should we stay or should we go: mechanisms and ecological consequences for biofilm dispersal. Nature Reviews. Microbiology 10, 39-50.

Methé, B.A., Nelson, K.E., Pop, M., et al, and the Human Microbiome Project Consortium, 2012. A framework for human microbiome research. Nature 486, 215-221.

Metwalli, K.H., Khan, S.A., Krom, B.P., Jabra-Rizk, M.A., 2013. Streptococcus mutans, Candida albicans, and the human mouth: a sticky situation. PLoS Pathogens 9, e1003616.

Nadell, C.D., Drescher, K., Foster, K.R., 2016. Spatial structure, cooperation and competition in biofilms. Nature Reviews. Microbiology $14,589-600$.
Nunan, N., 2017. The microbial habitat in soil: scale, heterogeneity and functional consequences. Journal of Plant Nutrition and Soil Science 180, 425-429.

O'Toole, G.A., Wong, G.C., 2016. Sensational biofilms: surface sensing in bacteria. Current Opinion in Microbiology 30, 139-146.

Oliveira, N.M., Martinez-Garcia, E., Xavier, J., Durham, W.M., Kolter, R., Kim, W., Foster, K.R., 2015. Biofilm formation as a response to ecological competition. PLoS Biology 13, e1002191.

Olsen, I., 2015. Biofilm-specific antibiotic tolerance and resistance. European Journal of Clinical Microbiology \& Infectious Diseases 34, 877-886.

Oppenheimer-Shaanan, Y., Steinberg, N., Kolodkin-Gal, I., 2013. Small molecules are natural triggers for the disassembly of biofilms. Trends in Microbiology 21, 594-601.

Or, D., Phutane, S., Dechesne, A., 2007. Extracellular polymeric substances affecting pore-scale hydrologic conditions for bacterial activity in unsaturated soils. Vadose Zone Journal 6, 298-305.

Pande, S., Kaftan, F., Lang, S., Svatoš, A., Germerodt, S., Kost, C., 2016. Privatization of cooperative benefits stabilizes mutualistic cross-feeding interactions in spatially structured environments. ISME Journal 10, 1413-1423.

Parsek, M.R., Greenberg, E.P., 2005. Sociomicrobiology: the connections between quorum sensing and biofilms. Trends in Microbiology 13, 27-33.

Patin, N.V., Duncan, K.R., Dorrestein, P.C., Jensen, P.R., 2016. Competitive strategies differentiate closely related species of marine actinobacteria. ISME Journal 10, 478-490.

Perez-Garcia, O.Lear, G., Singhal, N., 2016. Metabolic network modeling of microbial interactions in natural and engineered environmental systems. Frontiers in Microbiology 7, UNSP 673.

Raaijmakers, J.M., Mazzola, M., 2012. Diversity and natural functions of antibiotics produced by beneficial and plant pathogenic bacteria. Annual Review of Phytopathology 50, 403-424.

Raja, M., Hannan, A., Ali, K., 2010. Association of oral candidal carriage with dental caries in children. Caries Research 44, 272 276.

Raynaud, X., Nunan, N., 2014. Spatial ecology of bacteria at the microscale in soil. PLoS One 9, e87217.

Ren, D., Madsen, J.S., de la Cruz-Perera, C.I., Bergmark, L., Sørensen, S.J., Burmølle, M., 2014. High-throughput screening of multispecies biofilm formation and quantitative PCR-based assessment of individual species proportions, useful for exploring interspecific bacterial interactions. Microbial Ecology 68, 146-154.

Ren, D., Madsen, J.S., Sørensen, S.J., Burmølle, M., 2015. High prevalence of biofilm synergy among bacterial soil isolates in cocultures indicates bacterial interspecific cooperation. ISME Journal 9, 81-89.

Rillig, M.C., Muller, L.A., Lehmann, A., 2017. Soil aggregates as massively concurrent evolutionary incubators. ISME Journal 11, 1943-1948.

Rusconi, R., Garren, M., Stocker, R., 2014. Microfluidics expanding the frontiers of microbial ecology. Annual Review of Biophysics 43, 65-91.

Russel, J., Røder, H.L., Madsen, J.S., Burmølle, M., Sørensen, S.J., 2017. Antagonism correlates with metabolic similarity in diverse bacteria. Proceedings of the National Academy of Sciences of the 
United States of America 114, 10684-10688.

Schoustra, S.E., Dench, J., Dali, R., Aaron, S.D., Kassen, R., 2012.

Antagonistic interactions peak at intermediate genetic distance in clinical and laboratory strains of Pseudomonas aeruginosa. BMC Microbiology 12, 40.

Seneviratne, G., Jayasinghearachchi, H.S., 2005. A rhizobial biofilm with nitrogenase activity alters nutrient availability in a soil. Soil Biology \& Biochemistry 37, 1975-1978.

Sørensen, S.J., Bailey, M., Hansen, L.H., Kroer, N., Wuertz, S., 2005. Studying plasmid horizontal transfer in situ: a critical review. Nature Reviews. Microbiology 3, 700-710.

Szamosvári, D., Rütschlin, S., Böttcher, T., 2018. From pirates and killers: does metabolite diversity drive bacterial competition? Organic \& Biomolecular Chemistry 16, 2814-2819.

Sztajer, H., Szafranski, S.P., Tomasch, J., Reck, M., Nimtz, M., Rohde, M., Wagner-Döbler, I., 2014. Cross-feeding and interkingdom communication in dual-species biofilms of Streptococcus mutans and Candida albicans. ISME Journal 8, 2256-2271.
Trejo-Hernández, A., Andrade-Domínguez, A., Hernández, M., Encarnación, S., 2014. Interspecies competition triggers virulence and mutability in Candida albicans-Pseudomonas aeruginosa mixed biofilms. ISME Journal 8, 1974-1988.

Vetsigian, K., Jajoo, R., Kishony, R., 2011. Structure and evolution of Streptomyces interaction networks in soil and in silico. PLoS Biology 9, e1001184.

Vos, M., Wolf, A.B., Jennings, S.J., Kowalchuk, G.A., 2013. Microscale determinants of bacterial diversity in soil. FEMS Microbiology Reviews 37, 936-954.

Young, I.M., Crawford, J.W., 2004. Interactions and self-organization in the soil-microbe complex. Science 304, 1634-1637.

Young, I.M., Crawford, J.W., Nunan, N., Otten, W., Spiers, A., 2008. Chapter 4 Microbial Distribution in Soils. In: Advances in Agronomy. Elsevier, pp. 81-121.

Zengler, K., Zaramela, L.S., 2018. The social network of microorganisms - how auxotrophies shape complex communities. Nature Reviews. Microbiology 16, 383-390. 